\title{
Spatiotemporal Instability of Femtosecond Pulses in Graded-Index Multimode Fibers
}

\author{
Uğur Teğin ${ }^{(1)}$ and Bülend Ortaç
}

\begin{abstract}
We study the spatiotemporal instability generated by a universal unstable attractor in normal dispersion gradedindex multimode fiber for femtosecond pulses for the first time. Experimentally observed spatiotemporal instability sidebands are $91-\mathrm{THz}$ detuned from the pump wavelength of $800 \mathrm{~nm}$. Detailed analysis carried out numerically by employing coupledmode pulse propagation model. Numerically obtained results are well-aligned with experimental observations. Spatial evolution of the total field and spatiotemporal instability sidebands is calculated numerically, and for the input pulses of 200-fs duration, formation and evolution of spatiotemporal instability are shown in both spatial and temporal domains. Our results present the unique features of spatiotemporal instability, such as remarkable frequency shift with inherited beam shape of instability sidebands.
\end{abstract}

Index Terms-Ultra-short pulses, graded-index multimode fibers, nonlinear fiber optics, spatiotemporal pulse propagation.

\section{INTRODUCTION}

$\mathbf{N}$ OWADAYS, graded-index MMFs are attracting great interest. With the parabolic profile of their refractive index, these fibers provide novel features. In the last few years, researchers exploited these features and reported new nonlinear dynamics to explore such as spatiotemporal instability [1], [2], supercontinuum generation [3], [4], self-beam cleaning [3], [5], [6], multimode solitons [7] and their dispersive waves [8]. Among these effects, spatiotemporal instability, called also as geometric parametric instability (GPI) in the literature, excels as new wavelength generation technique since generated instability sidebands have remarkable frequency shift and inherit the spatial beam shape of the pump pulses [1], [2].

In 2003, Longhi's theoretical work predicted spatiotemporal instability effect in multimode fibers [9]. Because of the periodic refocusing of the beam, while propagating in gradedindex MMF, quasi-phase matching (between the pump, signal and idler) resulted as spatiotemporal instability sidebands and discrete peaks appear in the spectrum. In contrast to intermodal four-wave mixing which is capable of generating spectral peaks with the same amount of frequency shifts, spatiotemporal instability peaks inherit the spatial mode profile of the pump source [10]. Krupa et al. [1] reported the first

Manuscript received July 5, 2017; revised October 15, 2017; accepted October 28, 2017. Date of publication November 2, 2017; date of current version November 17, 2017. (Corresponding author: Uğur Teğin.)

The authors are with the National Nanotechnology Research Center (UNAM), Institute of Materials Science and Nanotechnology, Bilkent University, 06800 Ankara, Turkey (e-mail: ugur.tegin@bilkent.edu.tr; ortac@unam.bilkent.edu.tr).

Color versions of one or more of the figures in this letter are available online at http://ieeexplore.ieee.org.

Digital Object Identifier 10.1109/LPT.2017.2769343 experimental observation of spatiotemporal instability sidebands by using 900 picosecond pulses and observed sidebands are detuned more than $120 \mathrm{THz}$ from the pump frequency. Very recently, Wright et al. [2] studied the complex dynamics of the self-beam cleaning and spatiotemporal instability generation in graded-index MMF present theoretical model can explain the connection between self-beam cleaning and spatiotemporal instability by introducing an universal attractor model. The connection between these nonlinear phenomena is experimentally noticed by Lopez-Galmiche et al. [3]. while demonstrating of supercontinuum generation from GPI.

Aforementioned studies about on spatiotemporal instability focused on quasi-continuous pulse (hundred ps to few ns) evolution in graded-index MMF at normal dispersion regime due to the analogy between GPI and well-known modulation instability in single mode fibers presented by Longhi's theoretical work [9] thus spatiotemporal dynamics of femtosecond pulses are generally neglected. So far only self-beam cleaning effect in graded-index MMF is studied with femtosecond pulses [6]. In this letter, we present first observation of spatiotemporal instability of ultrashort pulses in graded-index MMF at normal dispersion. Our observation contradicts with Longhi's model which is not applicable to ultrashort pump pulses. Since self-beam cleaning with ultrashort pulses is presented by Liu et al. [6], our results validate the universal attractor model. Femtosecond, linearly polarized pulses at $800 \mathrm{~nm}$ start to experience spatiotemporal evolution inside $2.6 \mathrm{~m}$ graded-index MMF with $50 \mu \mathrm{m}$ core diameter and their evolution resulted as the generation of first spatiotemporal instability Stokes and anti-Stokes pair in the experiment. Observed instability sidebands appear in the spectrum as $91 \mathrm{THz}$ detuned with respect to launched pump pulse's central frequency. Formation and the broadening tendency of spatiotemporal instability sidebands with increasing launch pulse energy are experimentally reported. Spatial beam shape of first instability Stokes is measured and features Gaussian-like near-field beam profile. Numerical simulations confirm the experimental observations on the spatiotemporal instability and provide information on the spatial evolution of total field and sidebands inside the graded-index MMF. Simulation results provide detailed information on the generation and the formation behaviors of the instability sidebands. The positions of the sidebands in the frequency domain are within the reach of experimental observations.

\section{NUMERICAL STUDY}

To study the detailed evolution of femtosecond pulses in graded-index MMF numerical simulations are needed. 


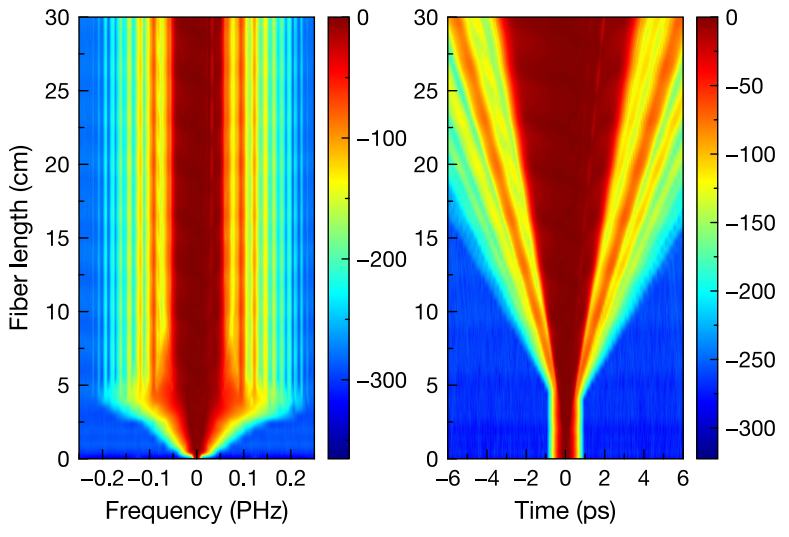

Fig. 1. Results from the numerical simulation showing total evolution through $30 \mathrm{~cm}$ fiber in frequency and time domains. The intensities in $\mathrm{dB}$ scale.

Pulse propagation in graded-index MMF can be simulated using the generalize multimode nonlinear Schödinger equation [11]-[13]. According to this model, the complex electric field can be expanded into a sum for modes $\mathrm{p}=0,1,2, \ldots$ and each mode can be represented with a transverse fiber mode profile. Evolution of temporal envelope of pth mode can be written as:

$$
\begin{aligned}
\frac{\partial A_{p}}{\partial z}= & i \delta \beta_{0}^{(p)} A_{p}-\delta \beta_{1}^{(p)} \frac{\partial A_{p}}{\partial t}-i \frac{\beta_{2}^{(p)}}{2} \frac{\partial^{2} A_{p}}{\partial^{2} t} \\
& +i \frac{\gamma}{3}\left(1+\frac{i}{\omega_{0}} \frac{\partial}{\partial t}\right) \sum_{l, m, n} \eta_{p l m n}\left[\left(1-f_{R}\right) A_{l} A_{m} A_{n}^{*}\right. \\
& \left.+f_{R} A_{l} \int h_{R} A_{m}(z, t-\tau) A_{n}^{*}(z, t-\tau) d \tau\right]
\end{aligned}
$$

where $\eta_{\text {plmn }}$ is nonlinear coupling coefficient, $f_{R} \approx 0.18$ is the fractional contribution of the Raman effect, $h_{R}$ is the delayed Raman response function and $\delta \beta_{0}^{(p)}\left(\delta \beta_{1}^{(p)}\right)$ is difference between first (second) Taylor expansion coefficient of propagation constant for corresponding and the fundamental mode. To solve Eq.(1) numerically, we use symmetrized splitstep Fourier method [14] and include Raman process and shock terms in our simulations.

A graded-index MMF with $50 \mu \mathrm{m}$ core diameter supports approximately 415 modes at $800 \mathrm{~nm}$ and simulating all of them will require complex and time-consuming calculations. Thus to achieve manageable computation times, we only consider first six zero-angular-momentum modes in our simulation. We launch pulses with 200 fs pulse duration, $350 \mathrm{~nJ}$ pulse energy at $800 \mathrm{~nm}$ which has a peak power considerably below the critical power for Kerr-induced self-focusing ( 2.44 MW) and this initials pulse energy is distributed among these six modes $(50 \%$ in $\mathrm{p}=0,18 \%$ in $\mathrm{p}=1,13 \%$ in $\mathrm{p}=2,10 \%$ in $\mathrm{p}=3,6 \%$ in $\mathrm{p}=4$ and $3 \%$ in $\mathrm{p}=5$ ). Propagation constants, dispersion and nonlinearity parameters are calculated as in [11]-[13]. We set $n_{0}$ as $1.4676, n_{2}$ as $2.7 \times 10^{-20} \mathrm{~m}^{2} / \mathrm{W}$, relative index difference as 0.01 , integration step as $10 \mu \mathrm{m}$, time window width as 15 ps with 2 fs resolution.

Spectral and temporal evolution of the pump pulse is first studied numerically for $30 \mathrm{~cm}$ graded-index MMF (Fig. 1). Femtosecond pulse starts to broaden in frequency domain
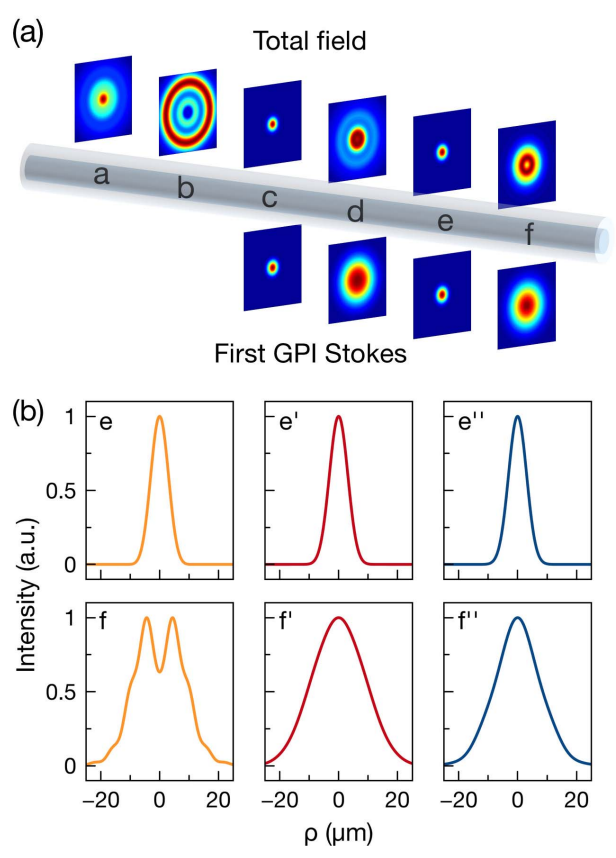

Fig. 2. Numerical results for spatial evolution inside the graded-index MMF with $50 \mu \mathrm{m}$ core diameter. (a) Spatial intensity distributions at $2 \mathrm{~cm}, 5 \mathrm{~cm}$, $17.03 \mathrm{~cm}, 17.12 \mathrm{~cm}, 17.21 \mathrm{~cm}, 17.28 \mathrm{~cm}$ (a-f). (b) Beam profiles of total field (e, f), first Stokes sideband (e', f') and first anti-Stokes sideband (e", f') for 17.21 and $17.28 \mathrm{~cm}$ of the fiber, respectively.

while propagating in the very first part of the gradedindex MMF. The observed relatively large spectral broadening is a unique feature of graded-index MMF and caused by high pump pulse energy [15], [16]. Numerical results indicate the generation of instability sidebands requires approximately 100 oscillations inside the graded-index MMF for our launch conditions and parameters (pulse duration, peak power and fiber core size etc.). Broadened pulse covers the emergence of instability sidebands and after a certain amount of propagation, discrete peaks become obviously visible. In numerical results, first pairs of instability sidebands appear at frequencies detuned approximately $90 \mathrm{THz}$ from the launched pump pulse frequency. First instability Stokes and anti-Stokes are centered around $1055 \mathrm{~nm}$ and $640 \mathrm{~nm}$, respectively. Simulation results indicate that after the formation is established, the intensity of instability sidebands starts to increase due to constant frequency generation with spatiotemporal propagation. At the end of the $30 \mathrm{~cm}$ fiber intensity difference between sidebands and the pump decreases down to $65 \mathrm{~dB}$. Along the fiber, the positions of the sidebands at frequency domains remains stable.

Obtaining the spatial evolution of the pulse inside the fiber is important in order to understand spatiotemporal changes. Thus we calculate the spatial evolution numerically at various positions inside the fiber and presented in Fig. 2 according to the simulation model [7], [12], [13]. Our results verify the spatial evolution of the beam experiences periodic refocusing along the graded-index MMF. This periodic behavior preserves the Gaussian-like spatial distribution for all focused points. We show the beam profile for three different focused points (Fig. 2(a).a, Fig. 2(a).c and Fig. 2(a).e). During the 


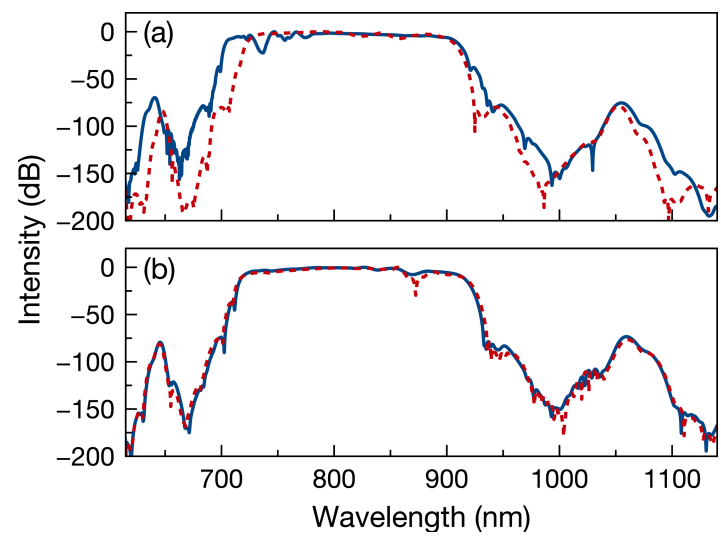

Fig. 3. Numerical spectra obtained from $30 \mathrm{~cm}$ graded-index MMF with different parameters. (a) Calculations with 6 cylindrically symmetric modes for different initial energy distributions (b) calculations with 3 cylindrically symmetric modes for different initial energy distributions.

spatiotemporal instability sideband generation $(\sim 5 \mathrm{~cm})$, we observed non-Gaussian intensity distribution for total field Fig. 2(a).b. After the instability sidebands emerge, spatial intensity distribution of total field approaches to Gaussian beam shape for spread points Fig. 2(a).d and Fig. 2(a).f as well. From numerical calculations, we also extract spatial intensity distribution of first instability Stokes and it indicates that inherited spatial intensity distribution is preserved at focused and spread points Fig. 2(a).c-f. This observation is a unique feature of the spatiotemporal evolution of the instability sidebands. In addition, we also studied spatial evolution of first instability anti-Stokes in different positions (focused and spread) along the graded-index MMF. As presented in Fig. 2(b), Gaussian-like spatial distributions are preserved for first instability anti-Stokes as well.

We study the effect of different launch conditions on instability sideband generation in the Fig.3(a). We compare above mentioned result (solid line) with the initial energy distribution between the modes as $30 \%$ in $\mathrm{p}=0,25 \%$ in $\mathrm{p}=1,15 \%$ in $\mathrm{p}=2,5 \%$ in $\mathrm{p}=3,3 \%$ in $\mathrm{p}=4$ and $2 \%$ in $\mathrm{p}=5$ (dashed line). Decreasing the energy of fundamental mode $(\mathrm{p}=0)$ results in less spectral broadening and slight frequency shift to first anti-Stokes sideband. Intensity difference between first instability sidebands between the different launch energy distribution is observed as $4 \mathrm{~dB}$. Next, we check the impact of considered number of modes in numerical studies. We run simulations with only first three zero-angular-momentum modes with different initial energy distributions. Obtained spectra for $30 \mathrm{~cm}$ graded-index MMF are presented in the Fig.3(b). First we distribute launch energy as $50 \%$ in $\mathrm{p}=0,30 \%$ in $\mathrm{p}=1$, $20 \%$ in $\mathrm{p}=2$ (solid line) then change as $35 \%$ in $\mathrm{p}=0,35 \%$ in $\mathrm{p}=1,30 \%$ in $\mathrm{p}=2$ (dashed line). Obtained spectra for these energy distributions present nearly identical features except intensity of generated sidebands. We notice that with increasing considered number of fiber modes higher conversion efficiency can be obtained.

\section{EXPERIMENTAL RESULTS AND DisCUSSIONS}

In the experiments, we use amplified Ti:Sapphire laser (Spitfire by Spectra-Physics) capable to generate linearly polarized, single-mode, 200 femtosecond ultrashort pulses

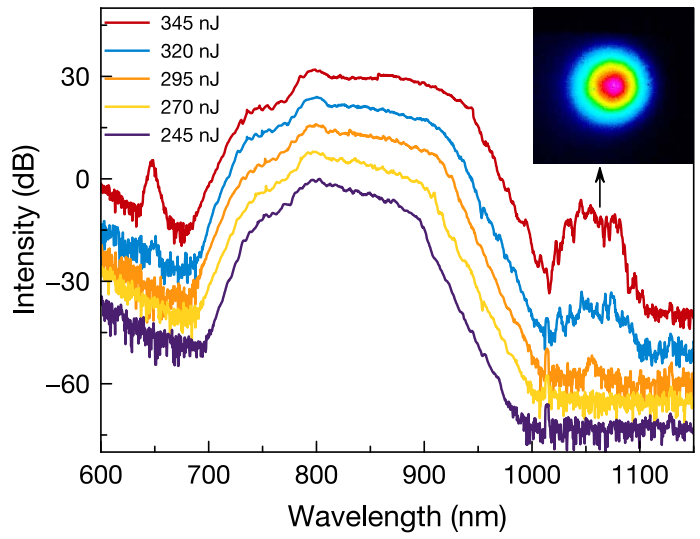

Fig. 4. Measured spectra as a function of launched pulse energy. Inset: near-filed beam profile of first Stokes sideband.

at $800 \mathrm{~nm}$ with $1 \mathrm{kHz}$ repetition rate for pump source. The fiber used in the experiment is a commercially available graded-index MMF (Thorlabs-GIF50C) with $50 \mu \mathrm{m}(125 \mu \mathrm{m})$ core (clad) diameter and 0.2 numerical aperture. We couple pump pulses into $2.6 \mathrm{~m}$ fiber with plano-convex lens and threeaxis translation stage configuration. We test various lenses with different focal lengths and obtain instability sideband generation in the measured spectrum with beam waists on fiber facet. For small beam waists, observed sidebands are unstable due to an environmental issue such as vibrations and degradation of optical alignment tools. On the other hand, selected $60 \mathrm{~mm}$ focal length lens provides $\sim 20 \mu \mathrm{m}$ waist size, thus excitation of higher-order modes is obtained easily and measured instability sidebands remain stable for several hours. In general, free space coupling efficiency greater than $80 \%$ could be achieved.

The generation and formation of experimentally observed instability sidebands are reported in detail Fig. 4 for different launched pulse energy conditions. We launch $200 \mathrm{fs}$ pump pulses at $800 \mathrm{~nm}$ with $\sim 10 \mathrm{~nm}$ FWHM into a $2.6 \mathrm{~m}$ graded-index MMF. Pump pulse experiences asymmetric spectral broadening for relatively low pulse energies (for example $270 \mathrm{~nJ}$ ). The observed asymmetric spectral broadening could be the result of stimulated Raman scattering (SRS). At high launched pulse energy $(345 \mathrm{~nJ})$, we observe further spectral broadening on pump region but discrete SRS peaks formation is not detected. With the increasing launched pulse energy for constant fiber length, first instability Stokes sideband emerges at $295 \mathrm{~nJ}$ launched pulse energy. Theory and simulation results indicate that both spatiotemporal instability sidebands should appear at the same time. Thus for launched pulse energy of $295 \mathrm{~nJ}$, first instability anti-Stokes should lie under the noise level of the optical spectrum analyzer. As launched pulse energy increases (at $320 \mathrm{~nJ}$ ), amplification and spectral broadening for first instability Stokes is recorded and in addition, first instability anti-Stokes also emerges. Similar spectral evolution (amplification and broadening) is also obtained for first anti-Stokes.

We successfully generate first instability peak pair with launched $345 \mathrm{~nJ}$ femtosecond pulses into graded-index MMF. First spatiotemporal instability peak pair is observed 


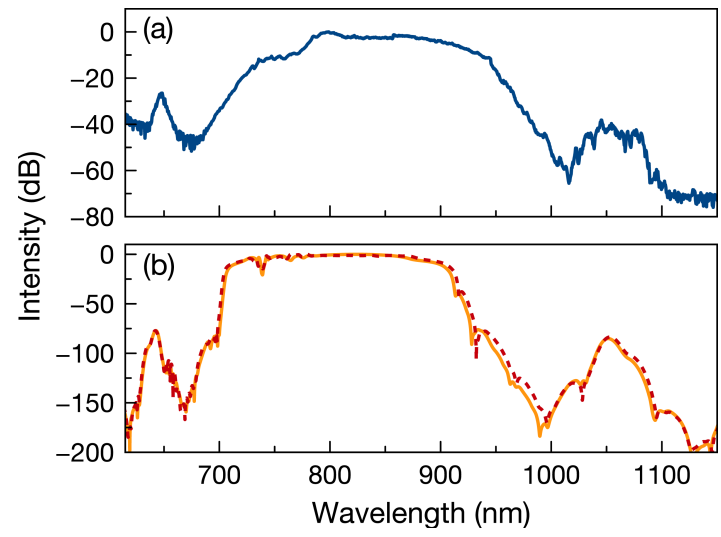

Fig. 5. Optical spectra obtained after propagating $2.6 \mathrm{~m}$ graded-index MMF. (a) Experimental measurement and (b) simulation results for different energy distribution between the modes.

with $\sim 91 \mathrm{THz}$ separation with respect to pump frequency $\left(f_{0}\right)$ (see Fig. 4(a)). First Stokes and anti-Stokes are centered around $1055 \mathrm{~nm}$ and $645 \mathrm{~nm}$, respectively. The corresponding optical spectrum bandwidth of first Stokes and anti-Stokes are $\sim 12 \mathrm{~nm}$ and $\sim 5 \mathrm{~nm}$, respectively. Even though bandwidths of instability sidebands seem different in wavelengths, in frequency domain they have approximately close bandwidths of $3.2 \mathrm{THz}$. We measure the near-field beam profile of the first Stokes sideband for launched pump pulses of $345 \mathrm{~nJ}$ pulse energy ( Fig.4-inset). To separate first Stokes from the pump pulse and the anti-Stokes, we use a longpass filter with $1000 \mathrm{~nm}$ cutoff wavelength. As expected from a spatiotemporal instability sideband, a clean (speckle free), Gaussian-like near-field beam profile is observed which is similar to the pump beam shape.

To compare obtained experimental result with our numerical model, we perform numerical simulations for $2.6 \mathrm{~m}$ gradedindex MMF (see Fig. 5). In order to decrease simulation time to manageable durations we simulate first three zeroangular-momentum modes with included Raman process and shock terms. First, we distribute $345 \mathrm{~nJ}$ pulse energy of the launched pulse to modes such as $50 \%$ in $\mathrm{p}=0,30 \%$ in $\mathrm{p}=1$ and $20 \%$ in $\mathrm{p}=2$ (solid-line). To check the effect of energy distribution between the modes we run simulations with $35 \%$ in $\mathrm{p}=0,35 \%$ in $\mathrm{p}=1$ and $30 \%$ in $\mathrm{p}=2$ distribution as well (dashed-line). For both distributions, positions and bandwidths of instability sidebands in frequency domain are similar to experimentally obtained results. We believe with increasing considered number of fiber modes more realistic results can be obtained from numerical simulations.

\section{CONCLUSION}

In conclusion, we study the spatiotemporal instability of ultrashort pulses in normal dispersion graded-index MMF. Our experimental results present spatiotemporal instability formation with femtosecond pump pulses first time in the literature and the reported spatiotemporal instability sidebands are well-aligned with numerical calculations. Detailed numerical studies revealed the generation and propagation behaviors of instability sidebands inside of MMF. Obtained results provide inside of the instability formation with ultrashort pulses to complete spatiotemporal pulse evolution studies and indicate that the attractor model reported for graded-index MMFs for also observable for ultrashort pump pulses [2]. For femtosecond pulses, self-beam cleaning is presented by Liu et al. [6] and our result complete the information gap for recently emerging research field. In future direction, with the intrinsic large frequency shift, spatiotemporal instability sidebands can be employed to generate new wavelengths for various application purposes.

\section{ACKNOWLEDGMENT}

The authors thank TUBITAK, TUBA-GEBIP, BAGEP, METU Prof. Dr. Mustafa Parlar Foundation and FABED for support and Ç. Şenel for insightful discussions.

\section{REFERENCES}

[1] K. Krupa et al., "Observation of geometric parametric instability induced by the periodic spatial self-imaging of multimode waves," Phys. Rev Lett., vol. 116, no. 18, p. 183901, 2016.

[2] L. G. Wright, Z. Liu, D. A. Nolan, M.-J. Li, D. N. Christodoulides, and F. W. Wise, "Self-organized instability in graded-index multimode fibres," Nature Photon., vol. 10, pp. 771-776, Nov. 2016.

[3] G. Lopez-Galmiche et al., "Visible supercontinuum generation in a graded index multimode fiber pumped at 1064 nm," Opt. Lett., vol. 41, no. 11, pp. 2553-2556, 2016.

[4] K. Krupa et al., "Spatiotemporal characterization of supercontinuum extending from the visible to the mid-infrared in a multimode gradedindex optical fiber," Opt. Lett., vol. 41, no. 24, pp. 5785-5788, 2016.

[5] K. Krupa et al., "Spatial beam self-cleaning in multimode fibres," Nature Photon., vol. 11, no. 4, pp. 237-241, Mar. 2017.

[6] Z. Liu, L. G. Wright, D. N. Christodoulides, and F. W. Wise, "Kerr self-cleaning of femtosecond-pulsed beams in graded-index multimode fiber," Opt. Lett., vol. 41, no. 16, pp. 3675-3678, 2016.

[7] W. H. Renninger and F. W. Wise, "Optical solitons in graded-index multimode fibres," Nature Commun., vol. 4, Apr. 2013, Art. no. 1719.

[8] L. G. Wright, D. N. Christodoulides, and F. W. Wise, "Controllable spatiotemporal nonlinear effects in multimode fibres," Nature Photon. vol. 9, no. 5, pp. 306-310, 2015.

[9] S. Longhi, "Modulational instability and space-time dynamics in nonlinear parabolic-index optical fibers," Opt. Lett., vol. 28, no. 23, pp. 2363-2365, 2003.

[10] E. Nazemosadat, H. Pourbeyram, and A. Mafi, "Phase matching for spontaneous frequency conversion via four-wave mixing in graded-index multimode optical fibers," J. Opt. Soc. Amer. B, Opt. Phys., vol. 33, no. 2, pp. 144-150, 2016.

[11] F. Poletti and P. Horak, "Description of ultrashort pulse propagation in multimode optical fibers," J. Opt. Soc. Amer. B, Opt. Phys., vol. 25, no. 10 , pp. 1645-1654, 2008.

[12] P. Horak and F. Poletti, "Multimode nonlinear fibre optics: Theory and applications," in Recent Progress in Optical Fiber Research. Rijeka, Croatia: InTech, 2012.

[13] A. Mafi, "Pulse propagation in a short nonlinear graded-index multimode optical fiber," J. Lightw. Technol., vol. 30, no. 17, pp. 2803-2811, Sep. 1, 2012.

[14] G. P. Agrawal, Nonlinear Fiber Optics. New York, NY, USA: Academic, 2007.

[15] J. T. Manassah, P. L. Baldeck, and R. R. Alfano, "Self-focusing and self-phase modulation in a parabolic graded-index optical fiber," Opt. Lett., vol. 13, no. 7, pp. 589-591, 1988.

[16] M. Karlsson, D. Anderson, and M. Desaix, "Dynamics of self-focusing and self-phase modulation in a parabolic index optical fiber,' Opt. Lett., vol. 17, no. 1, pp. 22-24, 1992. 